\title{
Analytical method of predicating the instabilities of a micro arch-shaped beam under electrostatic loading
}

\author{
Yin Zhang $\cdot$ Yisong Wang $\cdot$ Zhihong Li
}

Received: 7 April 2009/Accepted: 13 January 2010/Published online: 12 February 2010

(C) Springer-Verlag 2010

\begin{abstract}
An arch-shaped beam with different configurations under electrostatic loading experiences either the direct pull-in instability or the snap-through first and then the pull-in instability. When the pull-in instability occurs, the system collides with the electrode and adheres to it, which usually causes the system failure. When the snapthrough instability occurs, the system experiences a discontinuous displacement to flip over without colliding with the electrode. The snap-through instability is an ideal actuation mechanism because of the following reasons: (1) after snap-through the system regains the stability and capability of withstanding further loading; (2) the system flips back when the loading is reduced, i.e. the system can be used repetitively; and (3) when approaching snapthrough instability the system effective stiffness reduces toward zero, which leads to a fast flipping-over response. To differentiate these two types of instability responses for an arch-shaped beam is vital for the actuator design. For an arch-shaped beam under electrostatic loading, the nonlinear terms of the mid-plane stretching and the electrostatic loading make the analytical solution extremely difficult if not impossible and the related numerical solution is rather complex. Using the one mode expansion approximation and the truncation of the higher-order terms of the Taylor series, we present an analytical solution here. However, the
\end{abstract}

\section{Y. Zhang}

State Key Laboratory of Nonlinear Mechanics (LNM), Institute of Mechanics, Chinese Academy of Science, Beijing 100190, China

\section{Y. Wang · Z. Li $(\bowtie)$}

National Key Laboratory of Nano/Micro Fabrication

Technology, Institute of Microelectronics, Peking University,

Beijing 100871, China

e-mail: zhhli@ime.pku.edu.cn one mode approximation and the truncation error of the Taylor series can cause serious error in the solution. Therefore, an error-compensating mechanism is also proposed. The analytical results are compared with both the experimental data and the numerical multi-mode analysis. The analytical method presented here offers a simple yet efficient solution approach by retaining good accuracy to analyze the instability of an arch-shaped beam under electrostatic loading.

\section{Introduction}

The snap-through instability has been extensively explored and utilized in the micro-actuator and transducer design of arch (Ko et al. 2002; Qiu et al. 2004; Vangbo 1998; Zhang et al. 2007) and chevron/V-shaped structures (Hwang et al. 2003; Kugel et al. 1998; Sulfridge et al. 2002). For the arch and chevron/V-shaped structures as shown in Fig. 1, the bistable equilibrium configuration appears in certain loading region. The snap-through is the instability phenomenon that the system experiences a discontinuous displacement between the two separated stable equilibria. When the loading is the electrostatic type, the system will have another instability called pull-in (Francais et al. 1997; Nemirovsky and Bochobza-Degani 2001; Zhang et al. 2007). The mathematical analysis (Zhang et al. 2007) shows that when pull-in occurs, the system is also in a bistable state and tries to jump to the other stable equilibrium configuration, which is exactly the same scenario of snap-through. The only difference between the pull-in and snap-through instabilities is that for the pull-in instability the other equilibrium is physically unreachable unless the structure penetrates the electrode. As shown in Fig. 2 the consequence of pull-in is that the structure hits 
(a) Schematics and dimensions of the system

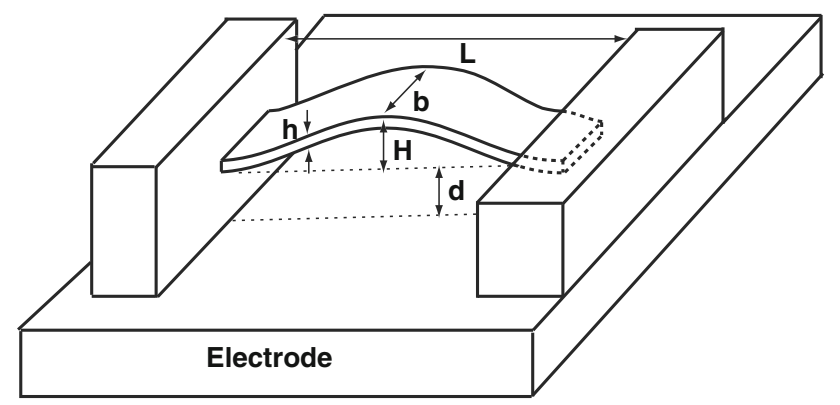

(b) Coordinate system

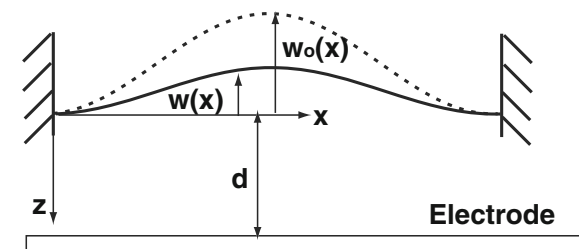

Fig. 1 a Dimensions of the system and $\mathbf{b}$ coordinate system

(a) Direct pull-in: beam hits the electrode and adheres to it when the instability occurs

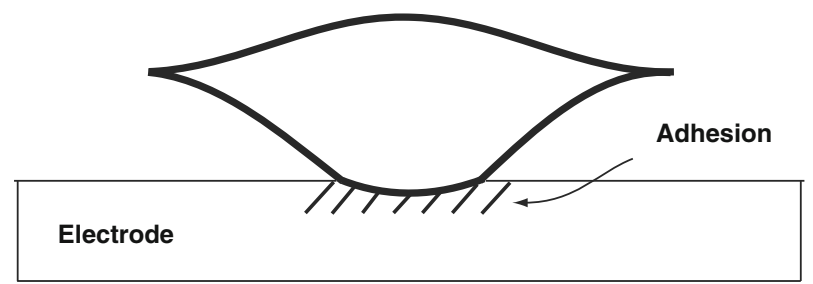

(b) Snap-through first: beam snaps-through first when the instability occurs; pull-in happens next with further increasing voltage.

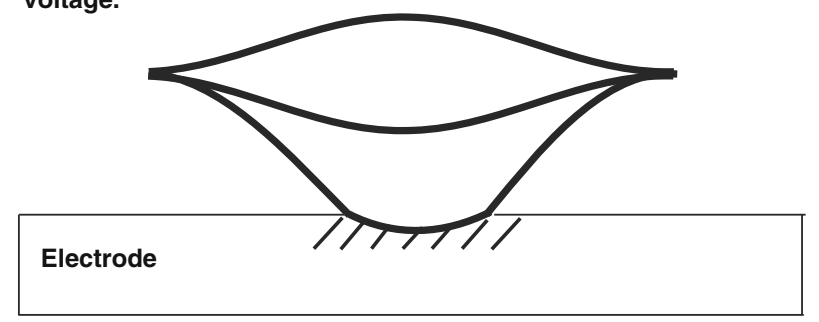

Fig. 2 Two response scenarios of the arch-shaped beam under electrostatic loading: a the beam has the direct pull-in instability and then adheres to the electrode after pull-in. b The beam snaps through first and then stays stable in a new equilibrium. The pull-in instability eventually occurs when further increasing electrostatic load

the electrode and adheres to it (Francais et al. 1997; Zhang et al. 2007). The snap-through and pull-in instabilities are the same type of instability termed as fold catastrophe (Thompson 1982), which in energy perspective involves the coalescence and extinction of a minimum and a maximum (Adams et al. 1998; Thompson 1982). Here, the pull-in instability is defined as the one that the structure collides with electrode and the snap-through instability is the one without collision.

The arch instability has been studied for quite some time (Fung and Kaplan 1952; Hsu 1967; Lock 1966). However, the recent researches (Bowden et al. 1998; Cerda 2005; Cerda et al. 1999, 2002; Cerda and Mahadevan 2003; Huang et al. 2007; Khang et al. 2006) reveal rich instability patterns in the large deformations of the beam, plate and shell structures. These instability researches have the wide applications in areas, such as dislocation (Cerda et al. 1999) and sheet crumpling (Cerda et al. 2002), wrinkling of thin film (Bowden et al. 1998; Huang et al. 2007), flexible electronics (Khang et al. 2006), skin wrinkles (Cerda and Mahadevan 2003), scar (Cerda 2005) and fingerprint formation (Kücken and Newell 2004). However, the solution to the nonlinear governing equation(s) of the above structures is "notoriously difficult" (Boudaoud et al. 2000). Although many new solution methods and analyses are proposed recently (Boudaoud et al. 2000; Cerda and Mahadevan 2003; Patrício et al. 1998; Pippard 1990), these new methods/analyses together with the old ones (Fung and Kaplan 1952; Hsu 1967; Lock 1966) by any standard are both difficult and formidable in terms of mathematical analysis and numerical solution. Furthermore, unlike those snap-through analyses (Hwang et al. 2003; Ko et al. 2002; Kugel et al. 1998; Qiu et al. 2004; Sulfridge et al. 2002; Vangbo 1998) the loading of which is displacementindependent, the electrostatic loading depends nonlinearly on the displacement. Therefore, besides the nonlinear term due to the mid-plane stretching of large deformation, the governing equation of an arch-shaped beam under electrostatic loading has an additional nonlinear term due to this electrostatic loading. The analysis in our previous work (Zhang et al. 2007), which adopts the old solution methods of using the multi-mode analysis and the numerical iteration is used here as a comparison example to illustrate the computation complexity and difficulty.

This paper is a follow-up to the previous work by Zhang et al. (2007). However, unlike the previous work of Zhang et al. (2007) which has a complex numerical solution, this work presents analytical method of solving the problem of an arch-shaped beam under electrostatic loading. The two key steps in this analytical method are: (1) to assume that the displacement consists of only one mode and (2) to expand the electrostatic loading term into the Taylor series up to the cubic order. The easiest way of computing this type of problem is to use the lumped-parameter method (Tilmans 1996), which converts the elastic-restoring continuum system into one or series of equivalent springs (Bochobza-Degani and Nemirovsky 2002; Francais et al. 1997; Nemirovsky and Bochobza-Degani 2001). 
The distributed- (or continuous-) parameter system has infinite degree of freedom (DOF) (Tilmans 1997). When lumping the continuous system into a one DOF system, the accuracy may be reduced and more lumped parameters are needed (Bochobza-Degani and Nemirovsky 2002; Gabby et al. 2000; Mehner et al. 2000). Besides accurately determining the equivalent spring stiffness, another technical difficulty of the lumped-parameter method for this particular problem is to determine the nonlinear stiffening parameter due to the beam mid-plane stretching, which can significantly change the system instability and dynamic properties (Bochobza-Degani and Nemirovsky 2002; Gabby et al. 2000; Mehner et al. 2000). The multimode analysis (Zhang et al. 2007) uses the exactly same lumping scheme as given by Tilmans (1997), which in essence is the modal decomposition method (Gabby et al. 2000; Mehner et al. 2000). The analytical method presented here is a compromise of the distributed-parameter method (Tilmans 1997). To achieve an analytical solution, the analytical method presented here gives up the infinite modes expansion scheme and keeps only one mode. In some loading regions of the snap-through and pull-in instability study, there are three equilibria under a fixed load. The numerical method like the multi-mode analysis (Zhang et al. 2007) has a great difficulty of solving all three equilibria. In addition, when studying the arch structure instability, it is very useful to construct a phase diagram which indicates the stability and instability regimes (Patrício et al. 1998; Pippard 1990). Those phase diagrams are made through a point-by-point scheme either by experiment (Pippard 1990) or by computation (Patrício et al. 1998). For the multi-mode analysis of the arch-shaped beam under electrostatic loading (Zhang et al. 2007) which has more control parameters than the mechanical loading case (Patrício et al. 1998; Pippard 1990), to construct such a phase diagram is a tremendous work because of the computation complexity. The advantages of the analytical method presented here include the following: the equivalent spring stiffness and stiffening parameter are easily determined, all three (possible) equilibria can easily be solved, the numerical computation cost is dramatically reduced, the definition of parameter $Q$ (see Sect. 2 for its definition) makes the work of telling the instability much easier.

However, in the analytical method the drawback of the Taylor series expansion approximation on the electrostatic loading term stands out when the displacement increases: the error becomes so large that the solution deviates from the real physical phenomenon. Also, the one mode expansion scheme is based on the assumption that the fundamental mode is the sole or dominant deflection shape. Under some conditions other modes can nevertheless participate considerably in the deflection, which contributes to the error, too. The above two error sources are systematically analyzed in this paper and a mechanism is also proposed to compensate the error of the Taylor series expansion due to the relatively large displacement. With the compensation mechanism the analytical method achieves a relatively good accuracy.

\section{Model development}

Figure 1a shows the schematic diagram of an arch-shaped beam with its two ends clamped and its related dimensions. $L, b$ and $h$ are the span, width and thickness of the beam, respectively. $d$ is the gap distance between the beam ends and electrode, and $H$ is the mid-span arch rise from the beam ends. The coordinate system is indicated in Fig. $1 \mathrm{~b}$. The following equation of equilibrium for an arch-shaped beam under a distributed load is adopted from Hsu (1967) and Lock (1966) with their dynamic terms truncated

$E^{*} I \frac{\mathrm{d}^{4}\left(w-w_{0}\right)}{\mathrm{d} x^{4}}+P \frac{\mathrm{d}^{2} w}{\mathrm{~d} x^{2}}+P_{0} \frac{\mathrm{d}^{2} w_{0}}{\mathrm{~d} x^{2}}+q(x)=0$.

$E^{*}$ is the effective Young's modulus of the beam. $I$ is the moment of inertia of the cross section and $I=b h^{3} / 12$ for a rectangular cross section. $w$ and $w_{0}$ are the deformed and initial coordinates of the beam centerline measured from the $x$-axis as shown in Fig. 1 b. $P$ is defined as $P=-P_{0}+$ $\frac{E^{*} A}{2 L} \int_{0}^{L}\left[\left(\frac{\mathrm{d} w_{0}}{\mathrm{~d} x}\right)^{2}-\left(\frac{\mathrm{d} w}{\mathrm{~d} x}\right)^{2}\right] \mathrm{d} x$ and $\frac{E^{*} A}{2 L} \int_{0}^{L}\left[\left(\frac{\mathrm{d} w_{0}}{\mathrm{~d} x}\right)^{2}-\left(\frac{\mathrm{d} w}{\mathrm{~d} x}\right)^{2}\right] \mathrm{d} x$ is the axial force due to the mid-plane stretching. The axial force induced by the mid-plane stretching is responsible for the stiffening effect (Bochobza-Degani and Nemirovsky 2002; Mehner et al. 2000). $A=b h$ is the cross section area. $P_{0}$ is the initial thrust and $P_{0}$ here has the opposite sign as that defined by Hsu (1967) in order to comply the rule that positive $P_{0}$ indicates tension and negative $P_{0}$ indicates compression (Abdel-Rahman et al. 2002). $q(x)$ is the distributed transverse load. Here, $q(x)$ is the electrostatic loading and $q=-\frac{\varepsilon b V^{2}}{2(d-w)^{2}} . \varepsilon$ is the dielectric constant of air and $V$ is the applied voltage. With the substitutions of $P$ and $q$, Eq. 1 is rewritten as the following:

$$
\begin{aligned}
& E^{*} I \frac{\mathrm{d}^{4}\left(w-w_{0}\right)}{\mathrm{d} x^{4}} \\
& \quad+\left\{-P_{0}+\frac{E^{*} A}{2 L} \int_{0}^{L}\left[\left(\frac{\mathrm{d} w_{0}}{\mathrm{~d} x}\right)^{2}-\left(\frac{\mathrm{d} w}{\mathrm{~d} x}\right)^{2}\right] \mathrm{d} x\right\} \frac{\mathrm{d}^{2} w}{\mathrm{~d} x^{2}} \\
& +P_{0} \frac{\mathrm{d}^{2} w_{0}}{\mathrm{~d} x^{2}}-\frac{\varepsilon b V^{2}}{2(d-w)^{2}}=0 .
\end{aligned}
$$

It is emphasized here that Eq. 2 is only valid for the shallow arch case. For a deep arch structure which experiences much larger defection, the elastica model is needed (Patrício et al. 1998; Pippard 1990). It is also worth mentioning the following two facts concerning the above 
governing equation: when the electrostatic force term of $\frac{\varepsilon b V^{2}}{2(d-w)^{2}}$ is set to zero, Eq. 2 recovers the governing equation describing the buckling and postbuckling of the microstructures with and without initial imperfections (Fang and Wickert 1994); when setting $w_{0}(x)=0$, Eq. 2 recovers the equation of equilibrium derived by AbdelRahman et al. (2002) for a flat beam under an electrostatic loading.

The following quantities are introduced to nondimensionalize Eq. 2:

$\xi=\frac{x}{L}, W_{0}(\xi)=\frac{w_{0}}{H+d}, W(\xi)=\frac{w}{H+d}, \Delta W(\xi)=\frac{\Delta w}{H+d}$.

$\Delta w$ is defined as $\Delta w=w(x)-w_{0}(x)$ (Zhang et al. 2007), which physically means the displacement from the initial configuration. $H$ and $d$ are the two major varying parameters for us to study the arch-shaped beam instability. With the above nondimensionalization scheme, once $\Delta W(\xi)=1$ we know that the beam collides with the electrode substrate and therefore the pull-in instability occurs.

The dimensionless equation of equilibrium can now be rewritten as follows:

$$
\begin{aligned}
& \frac{\mathrm{d}^{4} \Delta W}{\mathrm{~d} \xi^{4}}-\alpha_{1} \frac{\mathrm{d}^{2} \Delta W}{\mathrm{~d} \xi^{2}}-\alpha_{2} \int_{0}^{1}\left[2 \frac{\mathrm{d} \Delta W_{0}}{\mathrm{~d} \xi} \frac{\mathrm{d} \Delta W}{\mathrm{~d} \xi}-\left(\frac{\mathrm{d} \Delta W}{\mathrm{~d} \xi}\right)^{2}\right] \\
& \quad \times\left(\frac{\mathrm{d}^{2} W_{0}}{\mathrm{~d} \xi^{2}}+\frac{\mathrm{d}^{2} \Delta W}{\mathrm{~d} \xi^{2}}\right)-\frac{\alpha_{3} V^{2}}{\left[1 /\left(1+\alpha_{4}\right)-W_{0}-\Delta W\right]^{2}}=0 .
\end{aligned}
$$

$\alpha_{i} s(i=1-4)$ are defined as the following

$$
\begin{aligned}
& \alpha_{1}=\frac{P_{0} L^{2}}{E^{*} I}, \alpha_{2}=6\left(\frac{d+H}{h}\right)^{2}, \\
& \alpha_{3}=\frac{6 \varepsilon L^{4}}{E^{*} h^{3}(d+H)^{3}}, \alpha_{4}=\frac{H}{d} .
\end{aligned}
$$

The multi-modal analysis (Abdel-Rahman et al. 2002; Zhang et al. 2007) assumes the following expansion

$\Delta W=\sum_{i=1}^{N} a_{i} \phi_{i}(\xi)$.

$N$ is the mode number. $a_{i}$ is the constant modal amplitude to be determined. $\phi_{i}$ is the $i$ th mode shape of clampedclamped beam given by Chang and Craig (1969). For the details of using the multi-modal analysis to solve the archshaped beam instability problem, the reader should refer to the work by Zhang et al. (2007). Here, we do it differently by first expanding the function $f_{1}(\xi)=1 /\left[1 /\left(1+\alpha_{4}\right)-W\right]^{2}$ into the following Taylor series up to $\Delta W^{3}$ order:

$$
\begin{aligned}
& f_{1}(\xi)=\frac{1}{\left[1 /\left(1+\alpha_{4}\right)-W_{0}-\Delta W\right]^{2}} \\
& =\frac{1}{\left[1 /\left(1+\alpha_{4}\right)-W_{0}\right]^{2}-2\left[1 /\left(1+\alpha_{4}\right)-W_{0}\right] \Delta W-\Delta W^{2}} \\
& \approx f_{2}(\xi)=\frac{1}{\left[1 /\left(1+\alpha_{4}\right)-W_{0}\right]^{2}}+\frac{2 \Delta W}{\left[1 /\left(1+\alpha_{4}\right)-W_{0}\right]^{3}} \\
& \quad+\frac{3 \Delta W^{2}}{\left[1 /\left(1+\alpha_{4}\right)-W_{0}\right]^{4}}+\frac{4 \Delta W^{3}}{\left[1 /\left(1+\alpha_{4}\right)-W_{0}\right]^{5}}
\end{aligned}
$$

$f_{2}(\xi)$ is the Taylor series approximation of function $f_{1}(\xi)$ and the highest-order term kept in $f_{2}(\xi)$ is the cubic term, which as shown later in details is to obtain an analytical solution. As the Taylor series expansion truncates the higher-order terms, the error is thus generated. In general, the accuracy of the Taylor series expansion $f_{2}(\xi)$ reduces with the increase of $\left|\frac{\Delta W}{\left[1 /\left(1+\alpha_{4}\right)-W_{0}\right]}\right|$. The error function is defined as $\operatorname{erf}(\xi)=f_{1}(\xi)-f_{2}(\xi)$, which varies with different $\xi$. In our experiment, the arch is manufactured as an arc of a circle and for a shallow $\operatorname{arch} w_{0}(x)$ is approximated by a parabola as $w_{0}(x)=4 H\left(x^{2}-L x\right) / L^{2}$ (Zhang et al. 2007). Therefore, $W_{0}(\xi)=4 \alpha_{4}\left(\xi^{2}-\xi\right) /$ $\left(1+\alpha_{4}\right)$. Figure 3 shows the relative error of $\operatorname{erf}(\xi) / f_{1}(\xi)$ at $\xi=0.5$ as the function of the mid-span displacement, $\Delta W(0.5)$. Clearly, the error monotonously increases with the increasing displacement. It is clearly shown in Fig. 3 that when $\Delta W(0.5)=0.4$ the relative error is almost $10 \%$ and increases more dramatically with larger displacement. This error due to the Taylor series expansion as discussed later will lead to some physically unreasonable results. However, when the displacement is small, the Taylor series

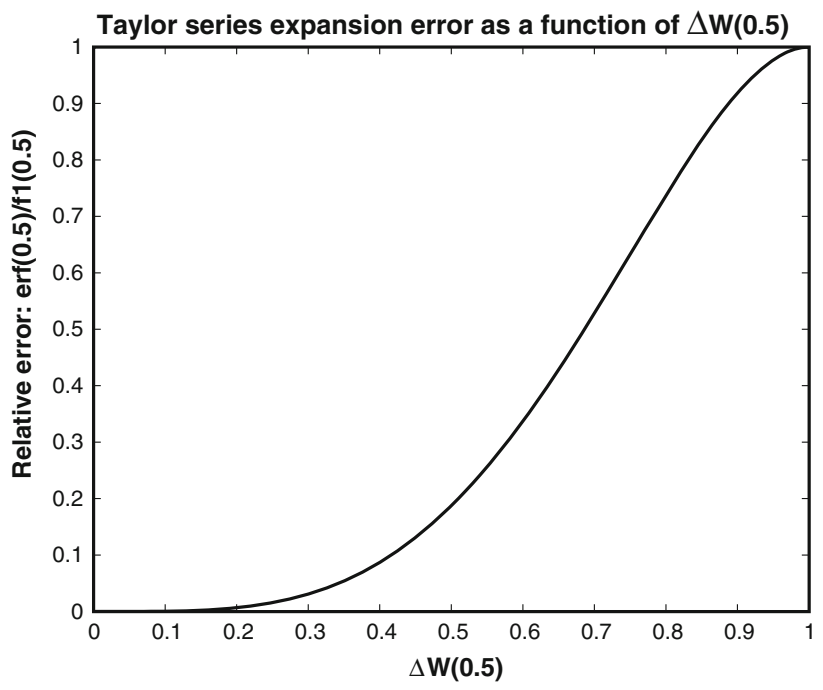

Fig. 3 The relative error due to the Taylor series expansion is shown as a function of the beam mid-span displacement of $\Delta W(0.5)$ 
expansion approximation is fairly accurate, for example, when $\Delta W(0.5)<0.35$ the relative error is less than $5 \%$. It is also noticed that in Fig. 3 the Taylor series expansion approximation of $f_{2}(\xi)$ is always smaller than $f_{1}(\xi)$.

For our analytical analysis, the following one mode expansion form is assumed

$\Delta W(\xi)=a_{1} \phi_{1}(\xi)$

Substitute both the Taylor series expansion approximation of Eq. 7 and the one mode expansion approximation of Eq. 8 into Eq. 4, times $\phi_{1}(\xi)$ and integrate from 0 to 1 , which is the Galerkin method of weighted residue (Meirovitch 1980). The following cubic equation is obtained:

$a_{1}^{3}+a_{s} a_{1}^{2}+b_{s} a_{1}+c_{s}=0$

It is noticed that the governing equation derived by the lumped parameter method also shares the above cubic equation form (Francais et al. 1997; Nemirovsky and Bochobza-Degani 2001). Here, the parameters $a_{s}, b_{s}$ and $c_{s}$ are defined as
$Q=\left(\frac{p}{3}\right)^{3}+\left(\frac{q}{2}\right)^{2}$

when $Q<0$, there are three real roots.

When $Q=0$, there are three real roots, at least two roots are the same.

When $Q>0$, there are one real root and two complex conjugate roots.

As shown later in Sect. 4, this $Q$ parameter is very useful for us to obtain the information about the instabilities. $a_{1}$ can be solved analytically by the procedure given by Korn and Korn (1968).

The validity of Eq. 9 relies on these two conditions: (1) the contribution of the other modes except the first mode to the equilibrium displacement is small (to be ignored); (2) the Taylor series expansion on the electrostatic loading is accurate. Although the other modes do contribute to the equilibrium configuration, their contribution as shown by the multi-modal analysis (Zhang et al. 2007) is relatively small. However, because the

$$
\begin{aligned}
a_{s} & =\frac{3 \alpha_{3} V^{2} \int_{0}^{1} \frac{\phi_{1}^{3}}{\left[1 /\left(1+\alpha_{4}\right)-W_{0}\right]^{4}} \mathrm{~d} \xi+\alpha_{2} \int_{0}^{1}\left(\phi_{1}^{\prime}\right)^{2} \mathrm{~d} \xi \int_{0}^{1} \phi_{1} W_{0}^{\prime \prime} \mathrm{d} \xi+2 \alpha_{2} \int_{0}^{1} W_{0}^{\prime} \phi_{1}^{\prime} \mathrm{d} \xi \int_{0}^{1} \phi_{1} \phi_{1}^{\prime \prime} \mathrm{d} \xi}{G} \\
b_{s} & =\frac{2 \alpha_{3} V^{2} \int_{0}^{1} \frac{\phi_{1}^{2}}{\left[1 /\left(1+\alpha_{4}\right)-W_{0}\right]^{3}} \mathrm{~d} \xi-\int_{0}^{1} \phi_{1} \phi_{1}^{\prime \prime \prime \prime} \mathrm{d} \xi+\alpha_{1} \int_{0}^{1} \phi_{1} \phi_{1}^{\prime \prime} \mathrm{d} \xi+2 \alpha_{2} \int_{0}^{1} W_{0}^{\prime} \phi_{1}^{\prime} \mathrm{d} \xi \int_{0}^{1} \phi_{1} W_{0}^{\prime \prime} \mathrm{d} \xi}{G} \\
c_{s} & =\frac{\alpha_{3} V^{2} \int_{0}^{1} \frac{\phi_{1}}{\left[1 /\left(1+\alpha_{4}\right)-W_{0}\right]^{2}} \mathrm{~d} \xi}{G} \\
G & =4 \alpha_{3} V^{2} \int_{0}^{1} \frac{\phi_{1}^{4}}{\left[1 /\left(1+\alpha_{4}\right)-W_{0}\right]^{5}} \mathrm{~d} \xi+\alpha_{2} \int_{0}^{1}\left(\phi_{1}^{\prime}\right)^{2} \mathrm{~d} \xi \int_{0}^{1} \phi_{1} \phi_{1}^{\prime \prime} \mathrm{d} \xi
\end{aligned}
$$

()$^{\prime}$ is defined as $\mathrm{d} / \mathrm{d} \xi$. Equation 9 is written in the following reduced form to have the standard Cardan solution (Korn and Korn 1968)

$b_{1}^{3}+p b_{1}+q=0$ arch-shaped beam may experience the snap-through and then stay stable in a new equilibrium, which is to say that the beam may have relatively large displacement, the second condition may not be satisfied. To compensate the error due to the Taylor series expansion, the parameter $\Lambda$ is introduced as follows

$\Lambda=\frac{\int_{0}^{1} \frac{\phi_{1}}{\left[1 /\left(1+\alpha_{4}\right)-W_{0}-a_{1} \phi_{1}\right]^{2}} \mathrm{~d} \xi}{\int_{0}^{1}\left\{\frac{\phi_{1}}{\left[1 /\left(1+\alpha_{4}\right)-W_{0}\right]^{2}}+\frac{2 a_{1} \phi_{1}^{2}}{\left[1 /\left(1+\alpha_{4}\right)-W_{0}\right]^{3}}+\frac{3 a_{1}^{2} \phi_{1}^{3}}{\left[1 /\left(1+\alpha_{4}\right)-W_{0}\right]^{4}}+\frac{4 a_{1}^{3} \phi_{1}^{4}}{\left[1 /\left(1+\alpha_{4}\right)-W_{0}\right]^{5}}\right\} \mathrm{d} \xi}$

$a_{1}=b_{1}-a_{s} / 3, p=-a_{s}^{2} / 3+b_{s}$ and $q=2\left(a_{s} / 3\right)^{3}$ $-a_{s} b_{s} / 3+c_{s}$. Here, $Q$ is introduced as a parameter indicating the cubic equation solution scenarios:
The purpose of defining the parameter $\Lambda$ in Eq. 13 is to have an overall weighted compensation for the error over the whole beam domain ( $\phi_{1}$ is the weighting function). 
Clearly, $\Lambda$ is a function of the modal amplitude (i.e. $\left.\Lambda=\Lambda\left(a_{1}\right)\right)$ and $\Lambda$ thus needs to be updated in each new equilibrium of different loading voltage. The compensated form of Eq. 9 can now be written as follows

$a_{1}^{3}+a_{s}^{*} a_{1}^{2}+b_{s}^{*} a_{1}+c_{s}^{*}=0$

The definitions of the new parameters are as follows eliminates the residual stress by a well-controlled process (Li et al. 2000).

The relative displacement of the arch-shaped beam with varying voltage is measured by MEMS Motion Analyzer apparatus (Zhang et al. 2007). In the system, a video camera and a frame grabber are utilized to record the MEMS device motion/deflection in real time. An integrated

$a_{s}^{*}=\frac{3 \Lambda \alpha_{3} V^{2} \int_{0}^{1} \frac{\phi_{1}^{3}}{\left[1 /\left(1+\alpha_{4}\right)-W_{0}\right]^{4}} \mathrm{~d} \xi+\alpha_{2} \int_{0}^{1}\left(\phi_{1}^{\prime}\right)^{2} \mathrm{~d} \xi \int_{0}^{1} \phi_{1} W_{0}^{\prime \prime} \mathrm{d} \xi+2 \alpha_{2} \int_{0}^{1} W_{0}^{\prime} \phi_{1}^{\prime} \mathrm{d} \xi \int_{0}^{1} \phi_{1} \phi_{1}^{\prime \prime} \mathrm{d} \xi}{G^{*}}$

$b_{s}^{*}=\frac{2 \Lambda \alpha_{3} V^{2} \int_{0}^{1} \frac{\phi_{1}^{2}}{\left[1 /\left(1+\alpha_{4}\right)-W_{0}\right]^{3}} \mathrm{~d} \xi-\int_{0}^{1} \phi_{1} \phi_{1}^{\prime \prime \prime \prime} \mathrm{d} \xi+\alpha_{1} \int_{0}^{1} \phi_{1} \phi_{1}^{\prime \prime} \mathrm{d} \xi+2 \alpha_{2} \int_{0}^{1} W_{0}^{\prime} \phi_{1}^{\prime} \mathrm{d} \xi \int_{0}^{1} \phi_{1} W_{0}^{\prime \prime} \mathrm{d} \xi}{G^{*}}$

$c_{s}^{*}=\frac{\Lambda \alpha_{3} V^{2} \int_{0}^{1} \frac{\phi_{1}}{\left[1 /\left(1+\alpha_{4}\right)-W_{0}\right]^{2}} \mathrm{~d} \xi}{G^{*}}$

$G^{*}=4 \Lambda \alpha_{3} V^{2} \int_{0}^{1} \frac{\phi_{1}^{4}}{\left[1 /\left(1+\alpha_{4}\right)-W_{0}\right]^{5}} \mathrm{~d} \xi+\alpha_{2} \int_{0}^{1}\left(\phi_{1}^{\prime}\right)^{2} \mathrm{~d} \xi \int_{0}^{1} \phi_{1} \phi_{1}^{\prime \prime} \mathrm{d} \xi$

\section{Fabrication and measurement}

The silicon glass anodic-bonding and deep etching release (SGADER) process (Xiao et al. 1999) is used to fabricate the testing device. The process to fabricate mechanical structures combines wafer bonding and deep reactive ion etching (DRIE) technologies. The starting materials are 4 inch medium doped silicon wafers and Pyrex 7740 glass wafers. First, a shallow trench about $10 \mu \mathrm{m}$ is etched by $\mathrm{KOH}$ (potassium hydroxide) to shape the anchors, which are used to sustain the movable beams and the electrodes. After the silicon dioxide is stripped by buffered HF (BHF), the silicon and glass wafers are then anodically bonded together under the conditions of $380^{\circ} \mathrm{C}$ and $-1,500 \mathrm{~V}$. The silicon wafers is thinned to about $50 \mu \mathrm{m}( \pm 5 \mu \mathrm{m})$ by $\mathrm{KOH}$ etching. Next, the beam and electrode structures are released through DRIE and a sputtered aluminum layer is used as a mask. After this step, the standard SGADER process is finished and the device is fabricated. The archshaped beam with an out-of-plane arch configuration is hard to be controlled using the planar microfabrication. Usually the arch can be formed by utilizing the mechanisms of the stress mismatch inside different layers for a multilayer structure or the stress gradients varying through the thickness of a homogeneous structure. However, in practice it is very difficult to control the beam parameters (for example, the arch rise $H$ and arch shape etc.) with high precision when utilizing the above mechanisms. Here, a bulk micromachining technology is used for the fabrication, which not only fabricates the beam with a high precision of beam dimensions by lithography, but also computer control and data acquisition unit, which consists of a variety of standard internal plug-in cards and external general purpose interface bus (GPIB) instruments, is used to supply power, generate signals, control voltages and measure the MEMS motion/deflection automatically. A micromanipulator system including a manual $\mathrm{X}-\mathrm{Y}-\mathrm{Z}$ micrometer position stage is used for positioning the devices in test. The hardware platform includes an optical microscope with general objectives and Mirau interferometer objectives, a stroboscopic laser source and a lead zirconate titanate (PZT) phase shifter for imaging. A software analysis package processes the captured images and signals, and generates testing reports. The in-plane dimension and motion measurement of MEMS devices are done by image matching of computer microvision, the outof-plane dimension and motion measurement MEMS devices are achieved by a two-beam microscopic interferometry with a five-step phase shifting.

\section{Results and discussions}

The beam is made of silicon with Young's modulus of $E=160 \mathrm{Gpa}$ and Poisson's ratio of $v=0.27$, the beam width $b$ is fixed as $b=50 \mu \mathrm{m}$, the initial thrust is $P_{0}=$ $-\frac{E^{*} A}{2 L} \int_{0}^{L}\left(\frac{\mathrm{d} w_{0}}{\mathrm{~d} x}\right)^{2} \mathrm{~d} x=-\frac{8 E^{*} b h H^{2}}{3 L^{2}}$, the dielectric constant of air is $\varepsilon=8.854 \times 10^{-12} \mathrm{~F} / \mathrm{m}$ (Zhang et al. 2007). Clearly, here the thrust $P_{0}$ does not include residual stress and its gradients which can have significant impact on the system equilibrium (Zhang and Zhao 2006) because of our residual stress- 
eliminating fabrication technique mentioned above (Li et al. 2000). As the beam width is relatively large, for a wide beam bending into a cylindrical surface the effective Young's modulus $E^{*}$ is taken as $E^{*}=E /\left(1-v^{2}\right)$ (Abdel-Rahman et al. 2002).

Figure 4 shows the results as given by the experimental measurements, the multi-modal analysis, one mode analysis of Eq. 9 and Eq. 14. The arch-shaped beam is with the dimensions of $L=475 \mu \mathrm{m}, H=2.3 \mu \mathrm{m}, d=3.4 \mu \mathrm{m}$ and $h=2.6 \mu \mathrm{m}$. The experimental and the multi-modal analysis (total five modes are used) data are taken from Zhang et al. (2007). For the convenience of statement, we introduce the state variable as $(V, \Delta W(0.5))$ (Zhang and Murphy 2005), $V$ is the applied voltage and $\Delta W(0.5)$ is the dimensionless beam mid-span displacement. The experiment indicates that the beam has a direct pull-in instability at $(75,0.1579)$, the multi-modal analysis indicates a direct pull-in at $(77.5,0.207)$ and Eq. 14 predicts a direct pull-in at $(79,0.1692)$. The criteria of the pull-in instability are simple: when $\Delta W(0.5) \geq 1$, pull-in occurs. The reasons for the difference between the experimental data and multimodal analysis are discussed in details by Zhang et al. (2007). The difference between the multi-modal analysis and Eq. 14 is mainly due to the single mode expansion of Eq. 8. One mode expansion eliminates all the other modes contribution to the equilibrium displacement. While, here the difference of the pull-in voltages predicated by the multi-mode analysis and Eq. 14 is less than 2\%. Although the difference of the critical mid-span displacements at pull-in predicated by the multi-mode analysis and Eq. 14 is around $18.3 \%$, the multi-mode analysis may have severe numerical fluctuation around the pull-in voltage (Zhang et al. 2007), which leads to inaccurate displacement predictions. When the system approaches the snap-through or the pull-in instability, the slope of the loading-displacement curve approaches infinity, which physically means the effective stiffness of the system is close to zero, or say, the system is on the verge of losing elastic restoring capability. Around the critical pull-in point tiny voltage/ loading change can induce very large displacement. In the multi-mode analysis of using Newton-Rhapson iteration method (Zhang et al. 2007), the Jacobian matrix becomes ill-conditioned around the snap-through or the pull-in point, which leads to the numerical fluctuation. To avoid or fix this numerical fluctuation problem is extremely difficult if not impossible. In contrast, Eq. 14 gives analytical solutions which provide the accurate critical voltage though the accuracy of the critical displacement is not guaranteed. In fact to derive the accurate critical displacement based on Eq. 1 is of no practical use. As around the pull-in instability or the snap-through instability the system effective stiffness as aforementioned is very small, any tiny variation of voltage loading will induce relatively large motion and the system inertial effects become significant or even predominant. Equation 1 is a static equation and a $70 \%$ displacement difference by the dynamic and static modelings is demonstrated by Lock (1966). In Fig. 4 the experimental data were obtained by increasing the loading voltage very slowly to reduce the dynamic effects as much as possible. In Fig. 4 the equilibrium solutions as given by Eq. 9 is also presented. When $62.5 \mathrm{~V} \leq V \leq 79 \mathrm{~V}$, Eq. 9 has three real roots, when $V<62.5 \mathrm{~V}$ or $V>79 \mathrm{~V}$ Eq. 9 has only one real root and two complex conjugate roots. Because the complex conjugate roots has no physical meaning in an equation of equilibrium like Eq. 9 or Eq. 14, physically the above solution scenario predicated by Eq. 9 means that the system either has three equilibria (two are stable and one is unstable) or single equilibrium, which is also the benchmark of telling an instability (Thompson 1982). Mathematically, $Q$ is the parameter which can be used to predicate the system instability. Here, it is worth pointing out that Eq. 14 also predicates the similar roots solution scenario to that predicated by Eq. 9. Just in order to avoid messy presentation in Figs. 4, 5, 6, 7, only one branch of the solutions as given by Eq. 14 is plotted. As shown in Fig. 4 the stable solution (marked by a solid line) collides the unstable solution (marked by a dashed line) at (79, 0.1692), at which $Q=0$. Further increasing voltage, $Q>0$ and the system experiences a qualitative change from the three equilibria state to the one equilibrium state and system experiences a discontinuous displacement jump, which is exactly the same as the snap-through of a shallow tied arch (Thompson 1982). When the triequilibria state appears, numerically solving the three equilibria is not an easy task. The multi-mode analysis by Zhang et al. (2007) made a de facto avoidance of seeking

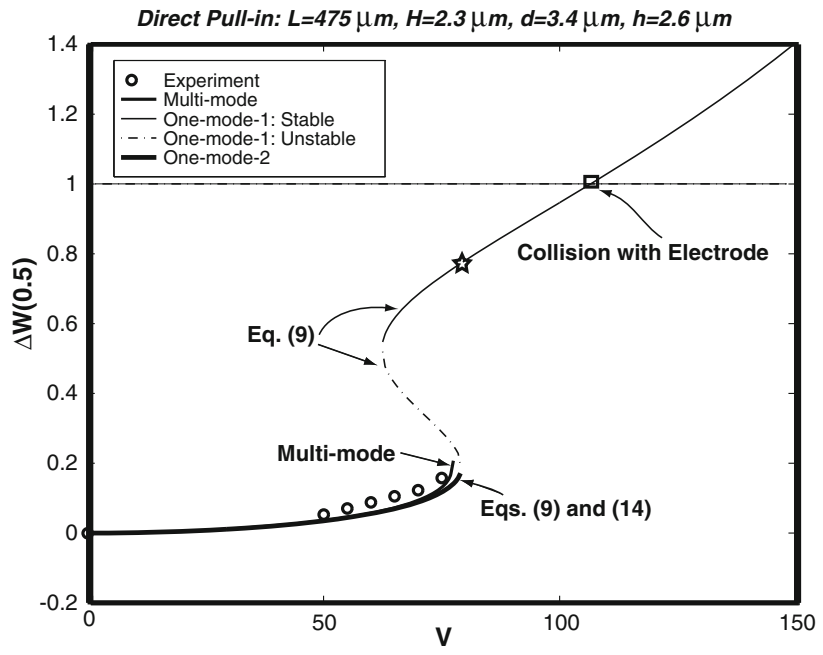

Fig. 4 The direct pull-in scenario with the beam dimensions of $L=475 \mu \mathrm{m}, H=2.3 \mu \mathrm{m}, d=3.4 \mu \mathrm{m}$ and $h=2.6 \mu \mathrm{m}$ 


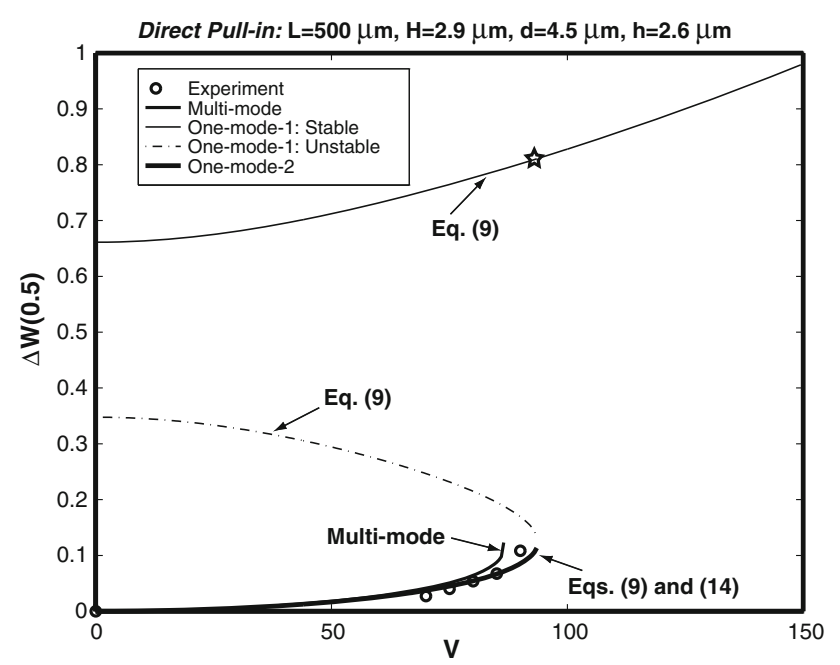

Fig. 5 The direct pull-in scenario with the beam dimensions of $L=500 \mu \mathrm{m}, H=2.9 \mu \mathrm{m}, d=4.5 \mu \mathrm{m}$ and $h=2.6 \mu \mathrm{m}$

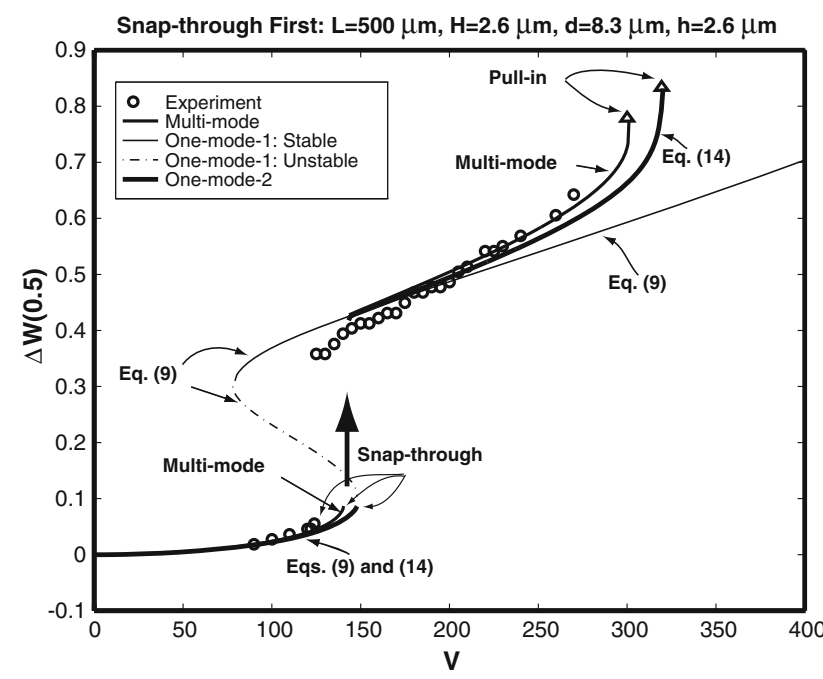

Fig. 6 The scenario of the beam experiencing both the snap-through and the pull-in instabilities. The beam dimensions are $L=500 \mu \mathrm{m}$, $H=2.6 \mu \mathrm{m}, d=8.3 \mu \mathrm{m}$ and $h=2.6 \mu \mathrm{m}$

the other two solutions. For the multi-mode analysis to have all three possible equilibrium solutions in certain region, not only a complex path-grabbing algorithm called pseudo-arclength method is required (Zhang and Murphy 2005) but also a good initial guess on the equilibrium configuration is needed (Patrício et al. 1998; Zhang and Murphy 2005). In contrast, it is easy for Eq. 9 or Eq. 14 to have all three equilibrium solutions when $Q<0$. However, Eq. 9 does not predict the pull-in instability at $V=79 \mathrm{~V}$. The experiment, multi-mode analysis and Eq. 14 all predicate that around the voltage range of 75-79 $\mathrm{V}$ the system experiences a discontinuous displacement jump and after the jump, all $\Delta W(0.5)>1$ (i.e. pull-in occurs). Equation 9 predicts the system jumps from $(79,0.1692)$ to $(79,0.7666)$

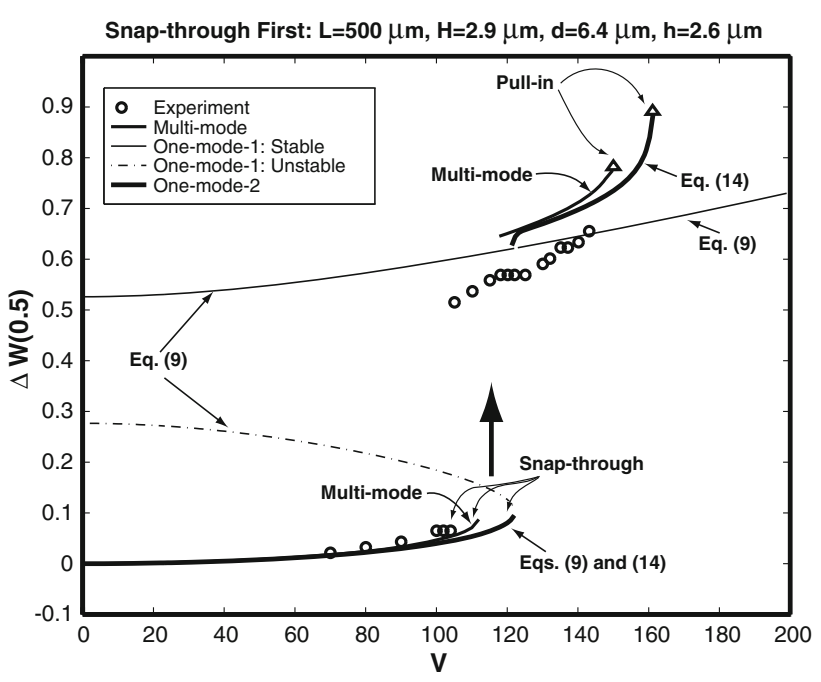

Fig. 7 The scenario of the beam experiencing both the snap-through and the pull-in instabilities. The beam dimensions are $L=500 \mu \mathrm{m}$, $H=2.9 \mu \mathrm{m}, d=6.4 \mu \mathrm{m}$ and $h=2.6 \mu \mathrm{m}$

(marked by a $\star$ in Fig. 4) and then the displacement keeps increasing continuously with the voltage increase. At (107, 1) (marked by a $\square$ in Fig. 4) the beam mid-span hits the electrode. Because of its large ratio of surface to volume, the micro-beam may adhere to the electrode. Once the adhesion occurs Eq. 1 can no longer be valid and new governing equation is needed (Mastrangelo and Hsu 1993; Zhang and Zhao 2004, 2005). When $V<79 \mathrm{~V}$, the curves as given by Eqs. 9 and 14 overlap each other. But after the jump, the two equations yield two quite different results and Eq. 9 leads to an erroneous one. The reason for that is explained by Fig. 3: before the jump $\Delta W(0.5)$ is small and the Taylor series expansion is accurate, after the jump $\Delta W(0.5)$ acquires a significant increase and the error due to the Taylor series expansion is thus significantly enlarged.

Figure 5 shows another direct pull-in scenario. The related dimensions in Fig. 5 are $L=500 \mu \mathrm{m}, H=2.9 \mu \mathrm{m}$, $d=4.5 \mu \mathrm{m}$ and $h=2.6 \mu \mathrm{m}$. Again the curves as given by Eqs. 9 and 14 overlap until $(93.5,0.1132)$. Equation 9 once again leads to an erroneous predication that the system jumps to $(93.5,0.812)$ marked by a $\star$ and then keeps a continuous increase. All the other three indicate the pull-in instability. The experiment indicates that the critical pull-in instability occurs at $(90,0.1081)$, the multi-mode analysis is at $(86.5,0.1234)$, Eqs. 9 and 14 are both at (93.5, 0.1132). The three equilibria state predicated by Eq. 9 in Fig. 5 starts from $V=0 \mathrm{~V}$ and ends at $V=93.5 \mathrm{~V}$.

Figure 6 shows the snap-through first and then pull-in scenario. The related dimensions are $L=500 \mu \mathrm{m}$, $H=2.6 \mu \mathrm{m}, d=8.3 \mu \mathrm{m}$ and $h=2.6 \mu \mathrm{m}$. The experiment indicates the snap-through from $(124,0.055)$ to $(124$, 0.3578), the multi-mode analysis has its snap-through from $(140.5,0.0868)$ to $(140.5,0.4203)$ and Eqs. 9 and 14 have 
their snap-through around $(148,0.086)$ to $(148,0.429)$. After the snap-through, if the voltage is decreased, the beam will lose its stability again at $(78,0.305)$ as predicated by Eq. 9 which is the intersection point of the solid and dotted lines in Fig. 6 and the beam will have a second snap-through to jump to $(78,0.0126)$. As seen in Fig. 6 , in the voltage range of $78 \leq V \leq 148$ (as predicated by Eq. 9), the beam has three equilibria in which two are stable (solid lines) and one is unstable (dashed line), i.e. the beam is in a bistable configuration. On the other hand, if the voltage is further increased after the beam first snapthrough, the pull-in instability occurs eventually. The experiment shows that the pull-in instability occurs at (270, 0.6442 ), the pull-in instability by the multi-mode analysis is at $(300,0.7615)$ marked by a $\Delta$, the pull-in instability by Eq. 14 is at $(327,0.843)$ marked by a $\Delta$. Again, Eq. 9 predicts the continuous increase and no pull-in after the snap-through. The error of the snap-through voltages as given by the multi-mode analysis and Eq. 14 is around 5\%. The error of the pull-in voltages as given by the multimode analysis and Eq. 14 is 9\%. It is noticed that in Fig. 6 the solutions scenario around the snap-through instability resembles that around the pull-in instability in Fig. 4, which further corroborates the aforementioned fact that the snap-through and pull-in are the same type of instability.

Figure 7 shows another snap-through first and then pullin scenario. The related dimensions are $L=500 \mu \mathrm{m}$, $H=2.9 \mu \mathrm{m}, d=6.4 \mu \mathrm{m}$ and $h=2.6 \mu \mathrm{m}$. The snapthrough by the experiment is from $(104,0.065)$ to $(104$, $0.505),(112,0.087)$ to $(112,0.6414)$ by the multi-mode analysis, around $(122,0.095)$ to $(122,0.62)$ by Eqs. 9 and 14 . Again, Eq. 9 does not predicate the pull-in instability after snap-through. The pull-in instability is indicated by the experiment at $(143,0.64),(150,0.771)$ by the multi-mode analysis (marked by a $\Delta)(163,0.893)$ by Eq. 14 (marked by a $\Delta$ ). The error study shows that there is approximately $9 \%$ difference for both the snap-through and pull-in voltages as given by the multi-mode analysis and Eq. 14, respectively. Once again, the resemblance of the solutions scenario around the snap-through instability in Fig. 7 to that around the pull-in instability in Fig. 5 is noticed.

\section{Summary}

An analytical method for the snap-through and the pull-in instabilities of an arch-shaped beam under electrostatic loading is presented. Because of the Taylor series expansion error, we introduce a compensated form of the Taylor series expansion on the electrostatic loading term and the comparison study is also presented. The uncompensated solution loses its accuracy with the increase of the displacement. The compensated solution gives relatively accurate results compared with both the experiments and multi-mode analysis. Physically two types of instability pattern are identified: the direct pull-in type, the snapthrough first and then pull-in type. Although the archshaped beam can have the above two different physical responses, the solution scenarios can be very similar. The parameter $Q$ defined above is the parameter which mathematically indicates the solution pattern and physically $Q=0$ indicates the critical instability point.

Acknowledgments Zhang is supported by the National Natural Science Foundation of China (NSFC, Grant 10721202) and the LNM initial funding for young investigators. Li is supported by the Foundation of National Key Laboratory of Nano/Micro Fabrication Technology.

\section{References}

Abdel-Rahman EM, Younis MI, Nayfeh AH (2002) Characterization of the mechanical behavior of an electrically actuated microbeam. J Micromech Microeng 12:759-766

Adams SG, Bertsch FM, Shaw KA, Hartwell PG, Moon FC, MacDonald NC (1998) Capacitance based tunable resonators. J Micromech Microeng 8:15-23

Bochobza-Degani O, Nemirovsky Y (2002) Modeling the pull-in parameters of electrostatic actuators with a novel lumped two degrees of freedom pull-in model. Sens Actuators A 97-98:569-578

Boudaoud A, Patrício P, Couder Y, Ben Amar M (2000) Dynamics of singularities in a constrained elastic plate. Nature 407:718-720

Bowden N, Brittain S, Evans AG, Hutchinson JW, Whitesides GM (1998) Spontaneous formation of ordered structures in thin films of metals supported on an elastomeric polymer. Nature 393:146-149

Cerda E (2005) Mechanics of scar. J Biomech 38:1598-1603

Cerda E, Mahadevan L (2003) Geometry and physics of wrinkling. Phys Rev Lett 90:074302

Cerda E, Chaleb S, Melo F, Mahadevan L (1999) Conical dislocations in crumpling. Nature 401:46-49

Cerda E, Rav-Chandar K, Mahadevan L (2002) Wrinkling of an elastic sheet under tension. Nature 419:579-580

Chang T, Craig RR Jr (1969) Normal modes of uniform beam. J Eng Mech 95:1027-1031

Fang W, Wickert JA (1994) Post buckling of micromachined beams. J Micromech Microeng 4:116-122

Francais O, Dufour I, Sarraute E (1997) Analytical static modelling and optimization of electrostatic micropumps. J Micromech Microeng 7:183-185

Fung YC, Kaplan A (1952) Buckling of low arches or curved beams of small curvatures. NACA Technical Note 2840

Gabby LD, Mehner JE, Senturia SD (2000) Computer-aided generation of nonlinear reduced order dynamic macromodels-I: non-stressstiffened case. J Microelectromech Syst 9:262-269

Hsu CS (1967) The effect of various parameters on the dynamic stability of a shallow arch. J Appl Mech 34:349-358

Huang J, Juszkiewicz M, de Jeu W, Cerda E, Emrick T, Menon N, Russell T (2007) Capillary wrinkling of floating thin poly film. Science 317:650-653

Hwang I, Shim Y, Lee J (2003) Modeling and experimental characterization of the chevron-type bi-stable microactuator. J Micromech Microeng 13:948-954

Khang D, Jiang H, Huang Y, Rogers JA (2006) A stretchable form of single-crystal silicon for high-performance electronics on rubber substrate. Science 311:208-212 
Ko JS, Lee ML, Lee D, Choi CA, Kim YT (2002) Development and application of a laterally driven electromagnetic microactuator. Appl Phys Lett 81:547-549

Korn GA, Korn TM (1968) Mathematical handbook for scientists and engineers, 2nd edn. Wiley, New York, pp 22-24

Kücken M, Newell AC (2004) A model for fingerprint formation. Europhys Lett 68:141-146

Kugel VD, Xu B, Zhang QM, Cross LE (1998) Bimorph-based piezoelectric air acoustic transducer: model. Sens Actuators A 69:234-242

Li Z, Xiao Z, Hao Y, Wu G, Wang Y (2000) A bulk micromachined vibratory lateral gyroscope fabricated with wafer bonding and deep trench etching. Sens Actuators A 83:24-29

Lock MH (1966) Snapping of a shallow sinusoidal arch under a step pressure load. AIAA J 4:1249-1256

Mastrangelo CH, Hsu CH (1993) Mechanical stability and adhesion of microstructures under capillary forces-part I: basic theory. J Microelectromech Syst 2:33-43

Mehner JE, Gabby LD, Senturia SD (2000) Computer-aided generation of nonlinear reduced order dynamic macromodels-II: stress-stiffened case. J Microelectromech Syst 9:270-278

Meirovitch L (1980) Computational methods in structural dynamics, chapter 8. Sijthoff \& Noordhoff, Rockville, Maryland

Nemirovsky Y, Bochobza-Degani O (2001) A methodology and model for the pull-in parameters of electrostatic actuators. J Microelectromech Syst 10:605-615

Patrício P, Adda-Bedia M, Ben Amar M (1998) An elastica problem: instabilities of an elastic arch. Physica D 124:285-295

Pippard AB (1990) The elastic arch and its modes of instability. Eur J Phys 11:359-365

Qiu J, Lang JH, Slocum AH (2004) A curved-beam bistable mechanism. J Microelectromech Syst 13:137-146
Sulfridge M, Saif MT, Miller N, O'Hara K (2002) Optical actuation of a bistable MEMS. J Microelectromech Syst 11:574-583

Thompson JMT (1982) Instabilities and catastrophes in science and engineering, chapter 1 and chapter 2. Wiley, New York

Tilmans HAC (1996) Equivalent circuit representation of electromechanical transducers: I. Lumped-parameter systems. J Micromech Microeng 6:157-176

Tilmans HAC (1997) Equivalent circuit representation of electromechanical transducers: II. Distributed-parameter systems. J Micromech Microeng 7:285-309

Vangbo M (1998) An analytical analysis of a compressed bistable buckled beam. Sens Actuators A 69:212-216

Xiao Z, Chen M, Wu G, Zhao C, Zhang D, Hao Y, Zhang G, Li Z (1999) Silicon microaccelerometer with $\mathrm{mg}$ resolution, high linearity and large frequency bandwidth fabricated with two mask bulk process. Sens Actuators A 77:113-119

Zhang Y, Murphy KD (2005) Secondary buckling and tertiary states of a beam on a non-linear elastic foundation. Int J Non Linear Mech 40:795-805

Zhang Y, Zhao Y (2004) Static study of cantilever beam stiction under electrostatic force influence. Acta Mechanica Solida Sinica 17:104-112

Zhang Y, Zhao Y (2005) Vibration of an adhered microbeam under a periodically shaking electrical force. J Adhes Sci Technol 19:799-815

Zhang Y, Zhao Y (2006) An effective method of determining the residual stress gradients in a micro-cantilever. Microsyst Technol 12:357-364

Zhang Y, Wang Y, Li Z, Huang Y, Li D (2007) Snap-through and pull-in instabilities of an arch-shaped beam under electrostatic loading. J Microelectromech Syst 16:684-693 